DOI: $10.2478 / \mathrm{v} 10324-012-0012-5$<smiles>CC1=C2CC2C1</smiles>

VERSITA
Analele Universităţii de Vest,

Timişoara

Seria Matematică - Informatică

L, 2, (2012), 15- 25

\title{
Modeling and Solving the Benchmark Case in a Differentiated Stackelberg Duopoly
}

\author{
Oana Ruxandra Tuns (Bode)
}

\begin{abstract}
A particular multilevel optimization problem, generated by a concrete economic problem related to technology transfer area, is approached in the present paper. We study the case when two firms compete on the market in a differentiated Stackelberg model and there is no technology transfer between the innovator firm (the leader firm) and the follower firm. A mathematical model is attached to this particular economic problem and an optimal solution is found. The model consists in a multilevel parametric programming problem, in which both the upper and the lower level functions are to be maximized under some given conditions.
\end{abstract}

AMS Subject Classification (2000). 90B50, 90C29, 90C31.

Keywords. Multilevel optimization, parametric optimization problem, benchmark, differentiated Stackelberg model.

\section{Introduction}

Licensing is one of the many significant methods used for technology transfer between firms. Licensing can be defined as the granting of permission to use intellectual property rights (such as patents, trademarks or technology) under defined conditions. As known, technology licensing represents a major economic activity and plays an important role for growth of firms and economy. Getting a new technology by technology licensing is a low risk access to 
increase the corporation profit. Although research and development (R\&D) is a good way to stimulate the growth of the firm profit, it needs not only to invest a lot of money, but also to spend a lot of time. Many firms have not enough capital or time to engage themselves in R\&D activity, so they choose to adopt a new technology and get a technology licensing $[2,3,4]$.

In [3] the authors have considered a differentiated Stackelberg model, when the leader firm engages itself in an R\&D process that gives an endogenous cost-reducing innovation. The authors have studied the licensing of the costreducing innovation in this paper.

In the present paper we study the case when in a differentiated Stackelberg duopoly model there is no technology transfer between the innovator firm and the follower firm (i.e. benchmark or pre-licensing case). A mathematical model is attached to this particular economic problem and an optimal solution is found. The model consists in a multilevel parametric optimization problem [1], in which both the upper and the lower level functions are to be maximized under some given conditions.

\section{The basic framework}

Let us consider a duopoly model where two firms, denoted by $F^{1}$ and $F^{0}$, produce $n$ differentiated goods. The inverse demand functions are given by $p^{h}=1-q^{h}-<d, q^{1-h}>$, where $p^{h}=\left(p_{1}^{h}, \ldots, p_{n}^{h}\right) \in \mathbb{R}^{n}$ represents the price of the firm $F^{h}, h=0,1 ; q^{h}=\left(q_{1}^{h}, \ldots, q_{n}^{h}\right) \in \mathbb{R}^{n}$ and $q^{1-h}=\left(q_{1}^{1-h}, \ldots, q_{n}^{1-h}\right) \in$ $\mathbb{R}^{n}$ represent the outputs of firms $F^{h}$ and $F^{1-h}$, respectively, where $h=1$; and $d$ represents the degree of the differentiation of goods, $d=\left(d_{1}, \ldots, d_{n}\right) \in \mathbb{R}^{n}$, with $d_{j} \in(0,1), \forall j \in\{1, \ldots, n\}$.

The duopoly market is modeled as a Stackelberg competition: the leader firm $F^{1}$ choose its output level and then the follower firm $F^{0}$ is free to choose its optimal output taking into account the leader's output; so, the firms do not decide simultaneously the level of their outputs. Initially, both firms have identical unit production $\operatorname{cost} c=\left(c_{1}, \ldots, c_{n}\right) \in \mathbb{R}^{n}$, with $c_{j} \in(0,1), \forall j \in$ $\{1, \ldots, n\}$. We consider that firm $F^{1}$, the leader firm, can engage itself in an $\mathrm{R} \& \mathrm{D}$ process in order to improve its technology. This allows a reduction of its production costs by an amount called innovation size. The cost-reducing innovation creates a new technology that reduces innovating firm's unit cost by the amount of $k$, while the amount invested in $R \& D$ is $k^{2} / 2$. We consider the case when there is no technology transfer between the innovator firm and the follower firm (i.e. benchmark or no-licensing case). 


\section{Modeling and solving the concrete economic pro- blem: Benchmark case}

Let $n \in \mathbb{N}^{*}$ be a natural number, $J=\{1, \ldots, n\}$, and $\gamma=\left(\gamma_{1}, \ldots, \gamma_{n}\right) \in \mathbb{R}^{n}$. Let $T \subseteq \mathbb{R}^{n}$ be the set of variation of the parameter $d=\left(d_{1}, d_{2}, \ldots, d_{n}\right) \in \mathbb{R}^{n}$. Using $d$ we can set the diagonal matrix $D \in \mathbb{R}^{n} \times \mathbb{R}^{n}$, such that

$$
D=\left(\begin{array}{cccc}
d_{1} & 0 & \cdots & 0 \\
0 & d_{2} & \cdots & 0 \\
\cdots & \cdots & \cdots & \cdots \\
0 & 0 & \cdots & d_{n}
\end{array}\right)
$$

For each $d=\left(d_{1}, d_{2}, \ldots, d_{n}\right) \in T$, let $f_{d}: \mathbb{R}^{n} \times \mathbb{R}^{n} \times \mathbb{R}^{n} \rightarrow \mathbb{R}, F_{d}: \mathbb{R}^{n} \times \mathbb{R}^{n} \times$ $\mathbb{R}^{n} \rightarrow \mathbb{R}$ and $g_{d}: \mathbb{R}^{n} \times \mathbb{R}^{n} \rightarrow \mathbb{R}$ be the functions given, respectively, by

$$
\begin{gathered}
f_{d}(x, y, z)=<\gamma-x-D y+z, x>, \forall(x, y, z) \in \mathbb{R}^{n} \times \mathbb{R}^{n} \times \mathbb{R}^{n}, \\
F_{d}(x, y, z)=<\gamma-x-D y+z, x>-\frac{1}{2}\|z\|^{2},
\end{gathered}
$$

i.e.

$$
F_{d}(x, y, z)=f_{d}(x, y, z)-\frac{1}{2}\|z\|^{2}, \forall(x, y, z) \in \mathbb{R}^{n} \times \mathbb{R}^{n} \times \mathbb{R}^{n}
$$

and

$$
g_{d}(x, y)=<\gamma-y-D x, y>, \forall(x, y) \in \mathbb{R}^{n} \times \mathbb{R}^{n} .
$$

For each $x \in \mathbb{R}_{+}^{n}$, let $S_{d}^{*}(x):=\operatorname{argmax}\left\{g_{d}(x, y) \mid y \in \mathbb{R}^{n}\right\}$. The elements of the set $S_{d}^{*}(x)$ will be generically denoted by $y^{x}$.

For each $z \in \mathbb{R}^{n}$, let $S_{d}^{*}(z):=\operatorname{argmax}\left\{f_{d}\left(x, y^{x}, z\right) \mid x \in \mathbb{R}_{+}^{n}\right\}$. The elements of the set $S_{d}^{*}(z)$ will be generically denoted by $x^{z}$.

Let us consider the three-level parametric programming problem

$$
(P ; T) \quad\left\{\begin{array}{l}
F_{d}(x, y, z) \rightarrow \max \\
y \in S_{d}^{*}(x) \\
x \in S_{d}^{*}(z) \\
z \in \mathbb{R}^{n}
\end{array} \quad, d \in T\right.
$$

For each $d \in T$, by $\left(P_{d}\right)$ we denote the three-level programming problem obtained from $(P ; T)$ if the parameter is fixed to $\mathrm{d}$.

Remark 1. If $T=] 0,1{ }^{n}$, then the problem $(P ; T)$ is the mathematical model attached to the basic economic problem described above. 


\subsection{Determining the set $S_{d}^{*}(x)$}

Let $d=\left(d_{1}, d_{2}, \ldots, d_{n}\right) \in T$ and $x \in \mathbb{R}_{+}^{n}$. We consider the problem

$$
\left(P_{d, x}^{1}\right) \quad\left\{\begin{array}{l}
\varphi_{d, x}(y) \rightarrow \max \\
y \in \mathbb{R}^{n}
\end{array}\right.
$$

where $\varphi_{d, x}(y)=g_{d}(x, y)=<\gamma-y-D x, y>, \forall y \in \mathbb{R}^{n}$.

We get that $\nabla \varphi_{d, x}(y)=-2 y+\gamma-D x, \forall y \in \mathbb{R}^{n}$. Since

$$
\nabla^{2} \varphi_{d, x}(y)=\left(\begin{array}{cccc}
-2 & 0 & \cdots & 0 \\
0 & -2 & \cdots & 0 \\
\cdots & \cdots & \cdots & \cdots \\
0 & 0 & \cdots & -2
\end{array}\right), \forall y \in \mathbb{R}^{n}
$$

it results that the function $\varphi_{d, x}$ is strictly concave. Hence, we get that

$$
y^{x}=\frac{1}{2}(\gamma-D x)
$$

is the maximum point of $\varphi_{d, x}$. Therefore, recalling the problem $\left(P_{d}\right), d \in T$, it results that for all $z \in \mathbb{R}^{n}$, if $S_{d}^{*}(z) \neq \emptyset$ and $x \in S_{d}^{*}(z)$, then the set $S_{d}^{*}(x)$ has just one element, i.e.

$$
S_{d}^{*}(x)=\left\{y^{x}\right\}=\left\{\frac{1}{2}(\gamma-D x)\right\} .
$$

\subsection{Determining the set $S_{d}^{*}(z)$}

Now, let $d=\left(d_{1}, d_{2}, \ldots, d_{n}\right) \in T$ and $z \in \mathbb{R}^{n}$. We consider the following programming problem

$$
\left(P_{d, z}^{2}\right) \quad\left\{\begin{array}{l}
\phi_{d, z}(x) \rightarrow \max \\
x \in \mathbb{R}_{+}^{n}
\end{array}\right.
$$

where $\phi_{d, z}(x)=f_{d}\left(x, y^{x}, z\right)=<\gamma-\frac{1}{2} D \gamma+z, x>+<\left(D^{2}-I_{n}\right) x, x>$, $\forall x \in \mathbb{R}_{+}^{n}, I_{n}$ being the identity matrix in $n$ dimensions.

We remark that

$$
\phi_{d, z}(x)=\sum_{j \in J}\left(-\left(1-\frac{d_{j}^{2}}{2}\right) x_{j}^{2}+\left(\gamma_{j}-\frac{d_{j} \gamma_{j}}{2}+z_{j}\right) x_{j}\right), \forall x \in \mathbb{R}^{n} .
$$

For $d \in T$ fixed, we denote by $J_{+}^{d}=\left\{j \in J \mid d_{j}^{2}>2\right\}, J_{-}^{d}=\left\{j \in J \mid d_{j}^{2}<2\right\}$, $J_{0}^{d}=\left\{j \in J \mid d_{j}^{2}=2\right\}$. 
For $d \in T$ and $z \in \mathbb{R}^{n}$ both fixed, we set $J_{0+}^{d}(z):=\left\{j \in J_{0}^{d} \mid \gamma_{j}-\frac{d_{j} \gamma_{j}}{2}+z_{j}>\right.$ $0\}, J_{00}^{d}(z):=\left\{j \in J_{0}^{d} \mid \gamma_{j}-\frac{d_{j} \gamma_{j}}{2}+z_{j}=0\right\}, J_{0-}^{d}(z):=\left\{j \in J_{0}^{d} \mid \gamma_{j}-\frac{d_{j} \gamma_{j}}{2}+z_{j}<\right.$ $0\}$.

Proposition 2. Let $d \in T$.

(i) If $J_{+}^{d} \neq \emptyset$, then the function $\phi_{d, z}$ is upper unbounded on $\mathbb{R}_{+}^{n}$, for each $z \in \mathbb{R}^{n}$;

(ii) If $J_{+}^{d}=\emptyset$ and $z \in \mathbb{R}^{n}$ such that $J_{0+}^{d}(z) \neq \emptyset$, then the function $\phi_{d, z}$ is upper unbounded on $\mathbb{R}_{+}^{n}$.

Proof. (i) Let us suppose that $J_{+}^{d} \neq \emptyset$ and let $h \in J_{+}^{d}$. Then $d_{h}^{2}>2$. If we consider the sequence $\left(x^{k}\right)_{k \geq 1}$, with $x^{k}=k e^{h}$ for each $k \in \mathbb{N}$, where $e^{h}$ represents the $h$-vector of the canonical base, i.e. $e^{h}=\left(e_{1}^{h}, e_{2}^{h}, \ldots, e_{n}^{h}\right) \in \mathbb{R}^{n}$ with

$$
e_{j}^{h}=\left\{\begin{array}{l}
0, \text { if } j \in J \backslash h, \\
1, \text { if } j=h
\end{array}\right.
$$

then, we have $x^{k} \in \mathbb{R}_{+}^{n}, \forall k \in N^{*}$ and

$$
\lim _{k \rightarrow \infty} \phi_{d, z}\left(x^{k}\right)=\lim _{k \rightarrow \infty}\left(-\left(1-\frac{d_{h}^{2}}{2}\right) k^{2}+\left(\gamma_{h}-\frac{d_{h} \gamma_{h}}{2}+z_{h}\right) k\right)=+\infty .
$$

Therefore, the function $\phi_{d, z}$ is upper unbounded on $\mathbb{R}_{+}^{n}$.

(ii) Now, let us suppose that $J_{+}^{d}=\emptyset$ and let $z \in \mathbb{R}^{n}$ such that $J_{0+}^{d}(z) \neq \emptyset$. Let $h \in J_{0+}^{d}(z)$. If we consider the sequence $\left(x^{k}\right)_{k \geq 1}$, with $x^{k}=k e^{h}$, for each $k \in \mathbb{N}$, where $e^{h}$ represents the $h$-vector of the canonical base, then $x^{k} \in \mathbb{R}_{+}^{n}$, for all $k \in \mathbb{R}$ and we have

$$
\lim _{k \rightarrow \infty} \phi_{d, z}\left(x^{k}\right)=\lim _{k \rightarrow \infty}\left(\gamma_{h}-\frac{d_{h} \gamma_{h}}{2}+z_{h}\right) k=+\infty .
$$

Therefore, the function $\phi_{d, z}$ is upper unbounded on $\mathbb{R}_{+}^{n}$.

Let $d \in T$ and $z \in \mathbb{R}^{n}$ be such that $J_{+}^{d}=\emptyset$ and $J_{0+}^{d}(z)=\emptyset$. Let us denote by $p=\operatorname{card}\left(J_{00}^{d}(z)\right)$ and $q=\operatorname{card}\left(J_{0-}^{d}(z)\right)$. Let $m=n-p-q=\operatorname{card}\left(J_{-}^{d}\right)$.

Remark 3. It is not difficult to see that, if $m=0$ and $\lambda=\left(\lambda_{1}, \ldots, \lambda_{n}\right) \in \mathbb{R}_{+}^{n}$, then $x^{z}=\left(x_{1}^{z}, \ldots, x_{n}^{z}\right) \in \mathbb{R}_{+}^{n}$, with

$$
x_{j}^{z}=\left\{\begin{array}{l}
0, \text { if } j \in J_{0-}^{d}(z) \\
\lambda_{j}, \text { if } j \in J_{00}^{d}(z),
\end{array}\right.
$$

is a maximum point of $\phi_{d, z}$. 
Under the hypothesis that $m>0$, let $J_{-}^{d}=\left\{j_{1}, \ldots, j_{m}\right\}$ be such that $1 \leq j_{1}<\cdots<j_{m} \leq n$. We consider the function $\tilde{\phi}_{d, z}: \mathbb{R}^{m} \rightarrow \mathbb{R}$ given by: $\forall\left(x_{j_{1}}, \ldots, x_{j_{m}}\right) \in \mathbb{R}^{m}$

$$
\tilde{\phi}_{d, z}\left(x_{j_{1}}, \ldots, x_{j_{m}}\right)=-\sum_{h=1}^{m}\left(1-\frac{d_{j_{h}}^{2}}{2}\right) x_{j_{h}}^{2}+\sum_{h=1}^{m}\left(\gamma_{j_{h}}-\frac{d_{j_{h}} \gamma_{j_{h}}}{2}+z_{j_{h}}\right) x_{j_{h}} .
$$

Proposition 4. The function $\tilde{\phi}_{d, z}: \mathbb{R}^{m} \rightarrow \mathbb{R}$ is strictly concave and its unique maximum point is $\tilde{x}=\left(\tilde{x}_{j_{1}}, \ldots, \tilde{x}_{j_{m}}\right)$, where

$$
\tilde{x}_{j_{h}}=\frac{2 \gamma_{j_{h}}-d_{j_{h}} \gamma_{j_{h}}+2 z_{j_{h}}}{2\left(2-d_{j_{h}}^{2}\right)}, \text { for each } h \in\{1, \ldots, m\} \text {. }
$$

Proof. It is easy to see that for each $h \in\{1, \ldots, m\}$ and $\forall\left(x_{j_{1}}, \ldots, x_{j_{m}}\right) \in \mathbb{R}^{m}$ we have

$$
\frac{\partial \tilde{\phi}_{d, z}}{\partial x_{j_{h}}}\left(x_{j_{1}}, \ldots, x_{j_{m}}\right)=-2\left(1-\frac{d_{j_{h}}^{2}}{2}\right) x_{j_{h}}+\gamma_{j_{h}}-\frac{d_{j_{h}} \gamma_{j_{h}}}{2}+z_{j_{h}}
$$

and

$$
\nabla^{2} \tilde{\phi}_{d, z}(x)=\left(\begin{array}{cccc}
-2+d_{j_{1}}^{2} & 0 & \cdots & 0 \\
0 & -2+d_{j_{2}}^{2} & \cdots & 0 \\
\cdots & \cdots & \cdots & \cdots \\
0 & 0 & \cdots & -2+d_{j_{m}}^{2}
\end{array}\right) .
$$

As $j_{h} \in J_{-}^{d}$ we have $-2+d_{j_{h}}^{2}<0$, for all $h \in\{1, \ldots, m\}$. Therefore, the function $\tilde{\phi}_{d, z}$ is strictly concave. Then, it has a unique maximum point given by $\left(\tilde{x}_{j_{1}}, \ldots, \tilde{x}_{j_{m}}\right)$. From (3) we get that $\tilde{x}_{j_{h}}=\frac{2 \gamma_{j_{h}}-d_{j_{h}} \gamma_{j_{h}}+2 z_{j_{h}}}{2\left(2-d_{j_{h}}^{2}\right)}$, for each $h \in\{1, \ldots, m\}$.

For $d \in T$ and $z \in \mathbb{R}^{n}$, let $J_{--}^{d}(z)=\left\{j \in J_{-}^{d} \mid \gamma_{j}-\frac{d_{j} \gamma_{j}}{2}+z_{j}<0\right\}$,

$J_{-+}^{d}(z)=\left\{j \in J_{-}^{d} \mid \gamma_{j}-\frac{d_{j} \gamma_{j}}{2}+z_{j}>0\right\}, J_{-0}^{d}(z)=\left\{j \in J_{-}^{d} \mid \gamma_{j}-\frac{d_{j} \gamma_{j}}{2}+z_{j}=0\right\}$.

We remark that $J_{-}^{d}=J_{--}^{d}(z) \cup J_{-+}^{d}(z) \cup J_{-0}^{d}(z)$.

From Proposition 4 and Remark 3, we obtain the following result. 
Corollary 5. If $d \in T$ and $z \in \mathbb{R}^{n}$ such that $J_{+}^{d}=\emptyset$ and $J_{0+}^{d}(z)=\emptyset$, then, for all $\lambda=\left(\lambda_{1}, \ldots, \lambda_{n}\right) \in \mathbb{R}_{+}^{n}$, the point $x^{z}=\left(x_{1}^{z}, \ldots, x_{n}^{z}\right)$, where

$$
x_{j}^{z}=\left\{\begin{array}{l}
\lambda_{j}, \text { if } j \in J_{00}^{d}(z), \\
0, \text { if } j \in\left(J_{0-}^{d}(z) \cup J_{--}^{d}(z) \cup J_{-0}^{d}(z)\right), \\
\frac{2 \gamma_{j}-d_{j} \gamma_{j}+2 z_{j}}{2\left(2-d_{j}^{2}\right)}, \text { if } j \in J_{-+}^{d}(z),
\end{array}\right.
$$

is a maximum point of the function $\phi_{d, z}$.

Based on the fact that for a multilevel optimization problem the objective functions of the lower level must have maximum (respectively, minimum) points, Proposition 2 implies that the set $T^{*}$ of feasibility of parameter $d$ has the following property

$$
T^{*} \subseteq\left\{d \in T \mid J_{+}^{d}=\emptyset \text { and } J_{0}^{d}=\emptyset\right\}=\left\{d \in T \mid d_{j}^{2}<2, \forall j \in J\right\} .
$$

In what follows, we consider that (5) holds. Under this hypothesis, for each $z \in \mathbb{R}$, the set $S_{d}^{*}(z)$ has exactly one element, i.e. we have $S_{d}^{*}(z)=\left\{x^{z}=\left(x_{1}^{z}, x_{2}^{z}, \ldots, x_{n}^{z}\right)\right\}$, where

$$
x_{j}^{z}=\left\{\begin{array}{l}
0, \text { if } j \in J_{--}^{d}(z) \cup J_{-0}^{d}(z), \\
\frac{2 \gamma_{j}-d_{j} \gamma_{j}+2 z_{j}}{2\left(2-d_{j}^{2}\right)}, \text { if } j \in J_{-+}^{d}(z) .
\end{array}\right.
$$

Let us remark that, if we consider $x_{j}^{z}$ as function of $z_{j}$, then this function is continuous on $\mathbb{R}$.

From (1) and (6), we obtain that

$$
y_{j}^{x_{j}^{z}}=\left\{\begin{array}{l}
\frac{\gamma_{j}}{2}, \text { if } j \in J_{--}^{d}(z) \cup J_{-0}^{d}(z), \\
\frac{\gamma_{j}\left(4-d_{j}^{2}\right)-2 d_{j}\left(\gamma_{j}+z_{j}\right)}{4\left(2-d_{j}^{2}\right)}, \text { if } j \in J_{-+}^{d}(z) .
\end{array}\right.
$$

Also, we remark that, if we consider $y_{j}^{x_{j}^{z}}$ as function of $z_{j}$, then this function is continuous on $\mathbb{R}$.

Under the hypothesis that (5) holds, we have $J=J_{-}^{d}$. Solving the initial problem $\left(P_{d}\right)$ is equivalent to determining the set

$$
\operatorname{argmax}\left\{F_{d}\left(x^{z}, y^{x^{z}}, z\right) \mid z \in \mathbb{R}^{n}\right\} .
$$

Therefore, now we solve the problem

$$
\left(P^{3}\right) \quad\left\{\begin{array}{l}
\theta_{d}(z) \rightarrow \max \\
z \in \mathbb{R}^{n}
\end{array}\right.
$$


where $\theta_{d}(z)=F_{d}\left(x^{z}, y^{x^{z}}, z\right)=<\gamma-x^{z}-D y^{x^{z}}+z, x^{z}>-\frac{1}{2}\|z\|^{2}$.

As $J=J_{-}^{d}$, we have $\theta_{d}(z)=\phi\left(x^{z}, y^{x^{z}}, z\right)-\frac{1}{2} \sum_{j \in J} z_{j}^{2}$ or

$\theta_{d}(z)=-\frac{1}{2} \sum_{j \in\left(J_{--}^{d}(z) \cup J_{-0}^{d}(z)\right)} z_{j}^{2}+\frac{1}{8} \sum_{j \in J_{-+}^{d}(z)} \frac{\left(2 \gamma_{j}-d_{j} \gamma_{j}+2 z_{j}\right)^{2}-4 z_{j}^{2}\left(2-d_{j}^{2}\right)}{2-d_{j}^{2}}$

Proposition 6. If there exists $j \in J$ such that:

(i) $2>d_{j}^{2}>1$ or

(ii) $d_{j}^{2}=1$ and $\gamma_{j} \neq 0$,

then the function $\theta_{d}$ is upper unbounded on $\mathbb{R}^{n}$.

Proof. (i) Let $s \in J$ be such that $2>d_{s}^{2}>1$. Let us consider the sequence $\left(z^{k}\right)_{k \in \mathbb{N}^{*}}$, where

$$
z_{j}^{k}=\left\{\begin{array}{l}
-\gamma_{j}+\frac{d_{j} \gamma_{j}}{2}, \text { if } j \in J \backslash\{s\} \\
-\gamma_{j}+\frac{d_{j} \gamma_{j}}{2}+k, \text { if } j=s
\end{array}\right.
$$

Then,

$$
\begin{gathered}
\theta_{d}\left(z^{k}\right)=-\frac{1}{2} \sum_{j \in J \backslash\{s\}}\left(-\gamma_{j}+\frac{d_{j} \gamma_{j}}{2}\right)^{2}+\frac{4 k^{2}-4\left(2-d_{s}^{2}\right)\left(-\gamma_{s}+\frac{d_{s} \gamma_{s}}{2}+k\right)^{2}}{8\left(2-d_{s}^{2}\right)}= \\
-\frac{1}{2} \sum_{j \in J \backslash\{s\}}\left(-\gamma_{j}+\frac{d_{j} \gamma_{j}}{2}\right)^{2}+ \\
\frac{4\left(d_{s}^{2}-1\right) k^{2}-4 k \gamma_{s}\left(2-d_{s}^{2}\right)\left(-2+d_{s}\right)-\left(2-d_{s}^{2}\right)\left(4 \gamma_{s}^{2}+d_{s}^{2} \gamma_{s}^{2}-4 \gamma_{s}^{2} d_{s}\right)}{8\left(2-d_{s}^{2}\right)}
\end{gathered}
$$

and $\lim _{k \rightarrow+\infty} \theta_{d}\left(z^{k}\right)=+\infty$. Therefore, the function $\theta_{d}\left(z^{k}\right)$ is upper unbounded on $\mathbb{R}^{n}$.

(ii) Let $s \in J$ be such that $d_{s}^{2}=1$ and $\gamma_{s} \neq 0$. If we consider the sequence $\left(z^{k}\right)_{k \in \mathbb{N}^{*}}$, with

$$
z_{j}^{k}=\left\{\begin{array}{l}
-\gamma_{j}+\frac{d_{j} \gamma_{j}}{2}, \text { if } j \in J \backslash\{s\} \\
-\gamma_{j}+\frac{d_{j} \gamma_{j}}{2}+k \operatorname{sgn}\left(\gamma_{j}\right), \text { if } j=s
\end{array}\right.
$$

then $\theta_{d}\left(z^{k}\right)=-\frac{1}{2} \sum_{j \in J \backslash\{s\}}\left(-\gamma_{j}+\frac{d_{j} \gamma_{j}}{2}\right)^{2}+\frac{1}{8}\left(4 k\left|\gamma_{s}\right|\left(2-d_{s}\right)-5 \gamma_{s}^{2}+4 d_{s} \gamma_{s}^{2}\right)$. Therefore, $\lim _{k \rightarrow+\infty} \theta_{d}\left(z^{k}\right)=+\infty$. It results that the function $\theta_{d}\left(z^{k}\right)$ is upper unbounded on $\mathbb{R}^{n}$. 
Remark 7. Proposition 6 implies that $T^{*} \subseteq\left\{d \in T \mid d_{j}^{2}<1, \forall j \in J\right\}$. Let $j \in J$. Let us consider $u_{j}: \mathbb{R} \rightarrow \mathbb{R}$, the function given by

$$
\begin{gathered}
u_{j}(t)=\left(2 \gamma_{j}-d_{j} \gamma_{j}+2 t\right)^{2}-4 t^{2}\left(2-d_{j}^{2}\right)= \\
=4 t^{2}\left(d_{j}^{2}-1\right)+4 \gamma_{j}\left(2-d_{j}\right) t+\gamma_{j}^{2}\left(2-d_{j}\right)^{2}, \forall t \in \mathbb{R} .
\end{gathered}
$$

It is easy to see that

$$
t^{*}=\frac{\gamma_{j}\left(2-d_{j}\right)}{2\left(1-d_{j}^{2}\right)}
$$

is a maximum point of $u_{j}$ on $\mathbb{R}$ and

$$
u_{j}\left(t^{*}\right)=\frac{\gamma_{j}^{2}\left(2-d_{j}\right)^{2}\left(2-d_{j}^{2}\right)}{1-d_{j}^{2}} \geq 0 .
$$

As $d_{j}^{2}<1, \forall j \in J$, it results that $u_{j}\left(t^{*}\right)=0$ if and only if $\gamma_{j}=0, \forall j \in J$.

Proposition 8. If $d \in T$ and $d_{j}^{2}<1, \forall j \in J$, then the function $\theta_{d}$ has an unique maximum point $z^{*}=\left(z_{1}^{*}, z_{2}^{*}, \ldots, z_{n}^{*}\right)$, where

$$
z_{j}^{*}=\frac{\gamma_{j}\left(2-d_{j}\right)}{2\left(1-d_{j}^{2}\right)}, \forall j \in J
$$

Proof. Based on (8) and (10), it results that the function $\theta_{d}$ will have the same maximum point as its restriction to the set

$$
\Lambda=\left\{z=\left(z_{1}, \ldots, z_{n}\right) \in \mathbb{R}^{n} \mid z_{j} \geq \frac{\gamma_{j}\left(d_{j}-2\right)}{2}, \forall j \in J\right\}
$$

For each $z \in \Lambda$, we have $\theta_{d}(z)=\frac{1}{8} \sum_{j \in J} \frac{\left(2 \gamma_{j}-d_{j} \gamma_{j}+2 z_{j}\right)^{2}-4 z_{j}^{2}\left(2-d_{j}^{2}\right)}{2-d_{j}^{2}}$.

On the set $\Lambda$, the function $\theta_{d}$ is twice differentiable.

We get that $\frac{\partial \theta_{d}}{\partial z_{j}}(z)=\frac{2 z_{j}\left(d_{j}^{2}-1\right)+2 \gamma_{j}-d_{j} \gamma_{j}}{2\left(2-d_{j}^{2}\right)}, \forall z \in \Lambda$, and

$$
\nabla^{2} \theta_{d}(z)=\left(\begin{array}{cccc}
\frac{d_{1}^{2}-1}{2-d_{1}^{2}} & 0 & \cdots & 0 \\
0 & \frac{d_{2}^{2}-1}{2-d_{2}^{2}} & \cdots & 0 \\
\cdots & \cdots & \cdots & \cdots \\
0 & 0 & \cdots & \frac{d_{n}^{2}-1}{2-d_{n}^{2}}
\end{array}\right), \forall z \in \mathbb{R}^{n}
$$


As $d_{j}^{2}<1, \forall j \in J$, it results that the function $\theta_{d}$ is strictly concave on $\Lambda$. Then, it has an unique maximum point.

As $\nabla \theta_{d}\left(z^{*}\right)=0_{n}$ for $z^{*}=\left(z_{1}^{*}, z_{2}^{*}, \ldots, z_{n}^{*}\right)$, where $z_{j}^{*}=\frac{\gamma_{j}\left(2-d_{j}\right)}{2\left(1-d_{j}^{2}\right)}, \forall j \in J$, and $z^{*} \in \Lambda$, it results that $z^{*}$ is the maximum point of $\theta_{d}$ on $\Lambda$, hence the maximum point of $\theta_{d}$ on $\mathbb{R}^{n}$.

Remark 9. From Proposition 8 we get that $\left\{d \in T \mid d_{j}^{2}<1, \forall j \in J\right\} \subseteq T^{*}$. Then, in view of Remark 7, we get that $T^{*}=\left\{d \in T \mid d_{j}^{2}<1, \forall j \in J\right\}$.

\section{Conclusions}

A three-level optimization problem provides the mathematical model of the economic problem approached in this paper. The originality of this problem is that it depends on the parameters $d$ and $\gamma$. For the particular case when $n=1, d \in] 0,1[$ and $\gamma \in] 0,1[$, the optimal solution of the problem coincide with the optimal solution obtained from the economic point of view presented in [3]. More, the result that the absolute value of parameter $d$ cannot exceed 1 has an important economic significance. Since $d$ denotes the degree of differentiation of goods, the result justifies the condition $d \in] 0,1[$, which is frequently used in the economic literature.

\section{Acknowledgements}

The author O. R. Tuns (Bode) wishes to thank for the financial support provided from program: Investing in people! Ph.D. scholarship, Project co-financed by the Sectoral Operational Program for Human Resources Development 2007 - 2013 Priority Axis 1. "Education and training in support for growth and development of a knowledge based society" Key area of intervention 1.5: Doctoral and post-doctoral programs in support of research. Contract POSDRU/88/1.5/S/60185 - "Innovative Doctoral Studies in a Knowledge Based Society", Babeş-Bolyai University, Cluj-Napoca, Romania. 


\section{References}

[1] St. Dempe, Foundations of Bilevel Programming, Kluwer Academic Publishers, 2002.

[2] F. A. Ferreira, Licensing in an International Competition with differentiated goods, Nonlinear Dynamics of Complex Systems: Application in Physical, Biological and Financial Systems, Editors: J.A. Tenreiro Machado, Dumitru Baleanu, Albert Luo. Springer Science + Business Media, (2011), 295-305.

[3] F. Ferreira and O. R. Bode, Licensing endogenous cost-reduction in a differentiated Stackelberg model, Communications in Nonlinear Science and Numerical Simulation, 18(2), (2013), 308-315.

[4] L. Filippini, Licensing contract in a Stackelberg model, The Manchester School, 73(5), (2005), 582-598.

Oana Ruxandra Tuns (Bode)

Faculty of Mathematics and Computer Science,

Babeş-Bolyai University,

1 M. Kogălniceanu Street, 400084 Cluj-Napoca,

Romania

E-mail: oanabode@yahoo.com

Received: 18.07.2012

Accepted: 14.09.2012 